\title{
Hallucinations in a Patient with Alzheimer's Disease During the COVID-19 Crisis: A Case Study
}

\author{
Mohamad El Haj, ${ }^{\mathrm{a}, \mathrm{b}, \mathrm{c}, *}$, Frank Larøi $\mathrm{i}^{\mathrm{d}, \mathrm{e}, \mathrm{f}}$ and Karim Gallouj ${ }^{\mathrm{b}}$ \\ ${ }^{a}$ Nantes Université, Univ Angers, Laboratoire de Psychologie des Pays de la Loire (LPPL - EA 4638), Nantes, \\ France \\ ${ }^{\mathrm{b}}$ Unité de Gériatrie, Centre Hospitalier de Tourcoing, Tourcoing, France \\ ${ }^{\mathrm{c}}$ Institut Universitaire de France, Paris, France \\ ${ }^{\mathrm{d}}$ Department of Biological and Medical Psychology, University of Bergen, Bergen, Norway \\ ${ }^{\mathrm{e}}$ Norwegian Center of Excellence for Mental Disorders Research, University of Oslo, Oslo, Norway \\ ${ }^{\mathrm{f}}$ Psychology and Neuroscience of Cognition Research Unit, University of Liège, Liège, Belgium
}

Accepted 1 October 2020

\begin{abstract}
While social distancing may be deemed necessary in order to avoid COVID-19 infections, the lockdown may impact mental health of patients with Alzheimer's disease (AD). We present a case study involving hallucinations in a patient with $\mathrm{AD}$ who lives in a nursing home during the COVID-19 crisis. We compared this patient's hallucination scores before and during the lockdown. We observed increased hallucinations during, compared to before, the lockdown. These increased hallucinations can be attributed to a number of elements such as the decreased in daily activities, social distancing, lack of physical contact with family members, and loneliness during the lockdown.
\end{abstract}

Keywords: Alzheimer's disease, COVID-19, hallucinations, social distancing

Because of their cognitive and functional impairments, patients with dementia are vulnerable at crisis, and this has been especially true during the coronavirus (COVID-19) pandemic. While COVID-19 has significantly turned our daily lives upside down, this impact is significantly pronounced in patients with dementia, especially those living in nursing homes [1]. Residents of nursing homes have a significant risk of COVID-19 infection. To cope with infection

\footnotetext{
*Correspondence to: Mohamad El Haj, Faculté de Psychologie, LPPL - Laboratoire de Psychologie des Pays de la Loire, Université de Nantes, Chemin de la Censive du Tertre, BP 81227, 44312 Nantes Cedex 3, France. E-mail: mohamad.elhaj@univ-nantes.fr.
}

and to limit its spread among residents, nursing homes in France (in addition to nursing homes in Europe and North America) have been obliged to prohibit physical contact between residents and families and friends and, in several cases, even between residents or between residents and caregivers. In France, lockdown in retirement homes was first announced on March 17, 2020. While the lockdown served to prevent infections, nursing homes were forced to separate residents physically from the outside world and to drastically reduce their activities. This lockdown was partially lifted on April 27, 2020 as residents were then allowed to receive visitations from one or two family members under strict conditions (e.g., short visits, only once per week). Because the 
lockdown may negatively impact cognitive and psychiatric function in Alzheimer's disease (AD), we present a case study of a patient with $A D$ who lives in a nursing home during the COVID-19 crisis. We show how this patient demonstrated an increase in hallucinations during the lockdown period.

A brief introduction of literature on hallucinations in $\mathrm{AD}$ is required before presenting the study case. While, at the cognitive level, AD is mainly characterized by memory decline [2], the disease is also characterized by psychiatric symptoms such as hallucinations $[3,4]$. The prevalence of hallucinations in $\mathrm{AD}$ can range from $4 \%$ to $76 \%$ (median $23 \%$ ) [5], and the occurrence of hallucinations can specifically be observed in patients with moderate or advanced AD [6]. Hallucinations in AD are mainly visual and auditory but olfactory, somatic, and tactile hallucinations can sometimes be observed [3]. Regardless of their modality, hallucinations are associated with greater cognitive impairment and a more rapidly deteriorating course in $\mathrm{AD}$ [7]. At the behavioral level, hallucinations in $\mathrm{AD}$ can lead to aggressive behavior [8], verbal outbursts [9], functional decline [10, 11], and falls [12]. In their turn, these behavioral consequences often lead to stress, depression, and a high level of burden of care in caregivers [13, 14].

Furthermore, it is reasonable to suppose that particular conditions, such as those during the COVID-19 crisis, may increase the occurrence of hallucinations in $\mathrm{AD}$ patients who are residents in nursing homes. This increased occurrence of hallucinations can occur because of a number of different elements, such as decreased daily activities, social distancing, and lack of physical contact with family members during the lockdown. We thus present a case study of a patient with moderate $\mathrm{AD}$ who had already reported hallucinations prior the COVID-19 crisis, and where an increase in the occurrence of hallucinations was observed during the lockdown.

\section{THE CASE STUDY}

Mr. B is 84 years of age, has had ten years of formal education, is right-handed, and is a native French speaker. Mr. B has been living in a nursing home in the region of Nantes (France) for nearly two years. His amnestic AD diagnosis was established five years ago based on NINCDS-ADRDA diagnostic criteria [2]. Mr. B was already participating in our ongoing study on hallucinations. On March 7, 2020, his hallucinations and cognitive ability were evaluated.
The cognitive evaluation included the Mini-Mental State Examination [15] on which Mr. B obtained a score of 16/30 points. This evaluation also included working memory (forward and backward spans) on which Mr. B did not retain more than three numbers on both the forward and backward spans.

\section{Hallucinations assessment and results}

We invited the caregiver (i.e., the nurse in charge of his ward) of Mr. B to evaluate his hallucinations on April 25, 2020. More specifically, we invited the caregiver to rate the following three items: "The patient sees objects or animals even though there is nothing there", "The patient is troubled by hearing voices in his head", "The patient declares that he hears a voice speaking aloud". These three items were used in previous studies involving hallucinations in $\mathrm{AD}$ [16-18]. The three items were assessed, during a phone interview, by the caregiver with the help of a five-point Likert scale ranging from zero ("certainly does not apply to Mr. B") to four (“certainly applies to him"). The maximum score was thus 12 points. Mr. B's score on the hallucinations assessment was 10 points. We compared this score to the score he obtained on March 7, 2020 when Mr. B's hallucinations were rated by the same caregiver, which was 7 points. Thus, the hallucinations score of Mr. B increased by three points from March 7, 2020 (before the lockdown) to April 25, 2020 (during the lockdown).

\section{DISCUSSION}

The present case study demonstrated an increase in the occurrence of hallucinations in a patient with AD during, compared to before, the lockdown. This increase can be attributed to several factors such as the social distancing, as implemented by the nursing home in which Mr. B is living. The nursing home where Mr. B resided (as did other facilities on a national level) implemented strict social distancing measures and drastic changes in the daily life of the residents from March 16, 2020 to April 27, 2020. Non-essential activities and services (e.g., hairdressers) were restricted, including basic social activities such as communal dining. Residents were invited not to leave their wards, and, when so, they were invited, as far as possible, to keep a safe distance from other residents in order to avoid contracting the virus. Critically, residents were not allowed to have any physical contact with their family members and 
friends. In the case of Mr. B, his wife used to visit him once or twice per week before the lockdown, but was not able to visit him during the lockdown. In addition, due to his cognitive decline, Mr. B was not able to use the current technology (e.g., Skype) to get in touch with his wife. The only contact occurred by phone, but probably only lasted a few minutes due to his cognitive decline.

While the (physical) social restrictions may be deemed necessary in order to avoid COVID-19 infections, these restrictions negatively impact cognitive and psychiatric function in patients with $\mathrm{AD}$. The decrease in social activities in the facilities and decrease in physical contact with family members may increase loneliness, itself leading to hallucinations. This assumption can be supported by research demonstrating how hallucinations in patients with $\mathrm{AD}$ can be associated with loneliness [17]. To compensate for boredom and emptiness, as this is associated with loneliness, patients with $\mathrm{AD}$ may generate internal stimuli to fulfil their need to communicate [17]. In other words, hallucinations in patients with $\mathrm{AD}$ can be regarded as a compensatory mechanism that aims to fulfil their communicatory needs [17].

The assumption that the social restrictions, as implemented during the COVID-19 crisis, may affect the mental health of patients with AD can be supported by two recent studies, the first study included patients living in retirement homes and the second study included patients living in their own homes. In the first study [19], patients with mild AD living in retirement homes were invited to rate their depression and anxiety during and before the COVID-19 crisis. Participants reported higher depression and anxiety during than before the COVID-19 crisis. The increased distress was attributed to the isolation of the residents and/or to the drastic changes in their daily life and to the care they receive in the retirement homes during the crisis. The effect of confinement on mental health of patients with $\mathrm{AD}$ was also observed in another study [20] on the effects of confinement during the COVID-19 crisis on neuropsychiatric symptoms in patients with advanced or mild AD who were confined in their own homes. In this study, caregivers were invited to report whether patients experienced increased neuropsychiatric symptoms during the confinement and, if it was the case, to rate this increase. Results demonstrated increased neuropsychiatric symptoms only in patients with advanced AD and this increased symptomatology significantly correlated with the duration of confinement.
A shortcoming of this study is the study case design. Although case studies are useful for generating research hypotheses, especially in unprecedented areas such as the effects of COVID-19 on neuropsychiatric symptoms in $\mathrm{AD}$, study case design do not have the level of methodological rigor to support the findings. Thus, the generalization of our findings should be viewed with caution. Research on a large scale is required to further investigate the effects of COVID-19 lockdown on hallucinations in patients with dementia living in nursing homes.

To summarize, while the lockdown during the COVID-19 crisis is necessary in order to prevent infection, this lockdown may negatively impact the wellbeing and mental health of patients [20]. The decrease in daily activities and social contact during the lockdown, as well as the physical separation of residents from loved ones, may lead to confusion, despair, and loneliness. Ultimately, the lockdown may increase hallucinations in residents, at least in those who were already experiencing hallucination prior to the lockdown.

\section{ACKNOWLEDGMENTS}

The study was supported by the LABEX (excellence laboratory, program investment for the future) DISTALZ (Development of Innovative Strategies for a Transdisciplinary Approach to Alzheimer Disease).

\section{CONFLICT OF INTEREST}

The authors declare no conflict of interest.

\section{REFERENCES}

[1] El Haj M, Allain P, Annweiler C, Boutoleau-Bretonnière C, Chapelet G, Gallouj K, Kapogiannis D, Roche J, Boudoukha $\mathrm{AH}$ (2020) Burnout of healthcare workers in acute care geriatric facilities during the COVID-19 crisis, an online-based study. J Alzheimers Dis, doi: 10.3233/JAD-201029.

[2] McKhann G, Knopman DS, Chertkow H, Hyman BT, Jack CR, Jr., Kawas CH, Klunk WE, Koroshetz WJ, Manly JJ, Mayeux R, Mohs RC, Morris JC, Rossor MN, Scheltens P, Carrillo MC, Thies B, Weintraub S, Phelps $\mathrm{CH}$ (2011) The diagnosis of dementia due to Alzheimer's disease: Recommendations from the National Institute on Aging-Alzheimer's Association workgroups on diagnostic guidelines for Alzheimer's disease. Alzheimers Dement 7, 263-269.

[3] El Haj M, Roche J, Jardri R, Kapogiannis D, Gallouj K, Antoine P (2017) Clinical and neurocognitive aspects of hallucinations in Alzheimer's disease. Neurosci Biobehav Rev 83, 713-720. 
[4] El Haj M, Badcock JC, Jardri R, Larøi F, Roche J, Sommer IE, Gallouj K (2019) A look into hallucinations: The relationship between visual imagery and hallucinations in Alzheimer's disease. Cogn Neuropsychiatry 24, 275-283.

[5] Bassiony MM, Lyketsos CG (2003) Delusions and hallucinations in Alzheimer's disease: Review of the brain decade. Psychosomatics 44, 388-401.

[6] Linszen MMJ, Lemstra AW, Dauwan M, Brouwer RM, Scheltens P, Sommer IEC (2018) Understanding hallucinations in probable Alzheimer's disease: Very low prevalence rates in a tertiary memory clinic. Alzheimers Dement (Amst) 10, 358-362.

[7] Weamer EA, Emanuel JE, Varon D, Miyahara S, Wilkosz PA, Lopez OL, Dekosky ST, Sweet RA (2009) The relationship of excess cognitive impairment in MCI and early Alzheimer's disease to the subsequent emergence of psychosis. Int Psychogeriatr 21, 78-85.

[8] Aarsland D, Cummings JL, Yenner G, Miller B (1996) Relationship of aggressive behavior to other neuropsychiatric symptoms in patients with Alzheimer's disease. Am J Psychiatry 153, 243-247.

[9] Lerner AJ, Koss E, Patterson MB, Ownby RL, Hedera P, Friedland RP, Whitehouse PJ (1994) Concomitants of visual hallucinations in Alzheimer's disease. Neurology 44, 523-527.

[10] Haupt M, Kurz A, Janner M (2000) A 2-year follow-up of behavioural and psychological symptoms in Alzheimer's disease. Dement Geriatr Cogn Disord 11, 147-152.

[11] Scarmeas N, Brandt J, Albert M, Hadjigeorgiou G, Papadimitriou A, Dubois B, Sarazin M, Devanand D, Honig L, Marder K, Bell K, Wegesin D, Blacker D, Stern Y (2005) Delusions and hallucinations are associated with worse outcome in Alzheimer disease. Arch Neurol 62, 1601-1608.

[12] Bassiony MM, Steinberg MS, Warren A, Rosenblatt A, Baker AS, Lyketsos CG (2000) Delusions and hallucinations in Alzheimer's disease: Prevalence and clinical correlates. Int J Geriatr Psychiatry 15, 99-107.
[13] Ornstein K, Gaugler JE, Devanand DP, Scarmeas N, Zhu C, Stern Y (2013) The differential impact of unique behavioral and psychological symptoms for the dementia caregiver: How and why do patients' individual symptom clusters impact caregiver depressive symptoms? Am J Geriatr Psychiatry 21, 1277-1286.

[14] Rocca P, Leotta D, Liffredo C, Mingrone C, Sigaudo M, Capellero B, Rocca G, Simoncini M, Pirfo E, Bogetto F (2010) Neuropsychiatric symptoms underlying caregiver stress and insight in Alzheimer's disease. Dement Geriatr Cogn Disord 30, 57-63.

[15] Folstein MF, Folstein SE, McHugh PR (1975) “Mini-mental state". A practical method for grading the cognitive state of patients for the clinician. J Psychiatr Res 12, 189-198.

[16] El Haj M, Gallouj K, Dehon H, Roche J, Laroi F (2018) Hallucinations in Alzheimer's disease: Failure to suppress irrelevant memories. Cogn Neuropsychiatry 23, 142-153.

[17] El Haj M, Jardri R, Laroi F, Antoine P (2016) Hallucinations, loneliness, and social isolation in Alzheimer's disease. Cogn Neuropsychiatry 21, 1-13.

[18] El Haj M, Laroi F, Gely-Nargeot MC, Raffard S (2015) Inhibitory deterioration may contribute to hallucinations in Alzheimer's disease. Cogn Neuropsychiatry 20, 281-295.

[19] El Haj M, Altintas E, Chapelet G, Kapogiannis D, Gallouj K (2020) High depression and anxiety in people with Alzheimer's disease living in retirement homes during the covid-19 crisis. Psychiatry Res 291, 113294.

[20] Boutoleau-Bretonnière C, Pouclet-Courtemanche H, Gillet A, Bernard A, Deruet AL, Gouraud I, Mazoue A, Lamy E, Rocher L, Kapogiannis D, El Haj M (2020) The effects of confinement on neuropsychiatric symptoms in Alzheimer's disease during the COVID-19 crisis. J Alzheimers Dis 76, 41-47. 